\title{
STRONG CONVERGENCE OF AN ITERATIVE METHOD FOR FINDING COMMON ZEROS OF A FINITE FAMILY OF ACCRETIVE OPERATORS
}

\author{
Jong SoO Jung
}

\begin{abstract}
Strong convergence theorems on viscosity approximation methods for finding a common zero of a finite family accretive operators are established in a reflexive and strictly Banach space having a uniformly Gâteaux differentiable norm. The main theorems supplement the recent corresponding results of Wong et al. [29] and Zegeye and Shahzad [32] to the viscosity method together with different control conditions. Our results also improve the corresponding results of $[9,16,18,19,25]$ for finite nonexpansive mappings to the case of finite pseudocontractive mappings.
\end{abstract}

\section{Introduction}

Let $E$ be a real Banach space and $C$ be a nonempty closed convex subset of $E$. Recall that a mapping $f: C \rightarrow C$ is a contraction on $C$ if there exists a constant $k \in(0,1)$ such that $\|f(x)-f(y)\| \leq k\|x-y\|, x, y \in C$. We use $\Sigma_{C}$ to denote the collection of mappings $f$ verifying the above inequality. That is, $\Sigma_{C}=\{f: C \rightarrow C \mid f$ is a contraction with constant $k\}$. Note that each $f \in \Sigma_{C}$ has a unique fixed point in $C$.

Now let $T: C \rightarrow C$ be a nonexpansive mapping (recall that a mapping $T: C \rightarrow C$ is nonexpansive if $\|T x-T y\| \leq\|x-y\|, \quad x, y \in C)$, and $F(T)$ denote the set of fixed points of $T$; that is, $F(T)=\{x \in C: x=T x\}$. $T$ is called pseudocontractive if there exists $j(x-y) \in J(x-y)$ such that

$$
\langle T x-T y, j(x-y)\rangle \leq\|x-y\|^{2} \text { for all } x, y \in C,
$$

where $J$ is the normalized duality mapping from $E$ to $2^{E^{*}}$. Clearly the class of nonexpansive mappings is a subset of the class of pseudocontractive mappings.

Received November 11, 2008; Revised January 21, 2009.

2000 Mathematics Subject Classification. 47H06, 47H10, 47J25, 49M05.

Key words and phrases. strong convergence, variational inequalities, nonexpansive mapping, fixed points, accretive operator, resolvent, sunny and nonexpansive retraction, strictly convex, uniformly Gâteaux differentiable norm.

This work was supported by the Korea Research Foundation Grant funded by the Korean Government (MOEHRD, Basic Research Promotion Fund) (KRF-2007-313-C00042). 
Closely related to the class of pseudocontractive mappings is the class of accretive operators. Recall that a (possibly multivalued) operator $A \subset E \times$ $E$ with the domain $D(A)$ and the range $R(A)$ in $E$ is accretive if, for each $x_{i} \in D(A)$ and $y_{i} \in A x_{i}(i=1,2)$, there exists a $j \in J\left(x_{1}-x_{2}\right)$ such that $\left\langle y_{1}-y_{2}, j\right\rangle \geq 0$. (Here $J$ is the duality mapping.) An accretive operator $A$ is said to satisfy the range condition if $\overline{D(A)} \subset R(I+r A)$ for all $r>0$. An accretive operator $A$ is $m$-accretive if $R(I+r A)=E$ for each $r>0$. If $A$ is an accretive operator which satisfies the range condition, then we can define, for each $r>0$ a mapping $J_{r}: R(I+r A) \rightarrow D(A)$ defined by $J_{r}=(I+r A)^{-1}$, which is called the resolvent of $A$. We know that $J_{r}$ is nonexpansive singlevalued mapping and $F\left(J_{r}\right)=A^{-1} 0$ for all $r>0$. The set of zero of $A$ is denoted by $N(A)$, that is,

$$
N(A):=\{x \in D(A): 0 \in A x\}=A^{-1} 0 .
$$

If $A^{-1} 0 \neq \emptyset$, then the inclusion $0 \in A x$ is solvable. We also observe that $x$ is a zero of the accretive operator $A$ if and only if it is a fixed point of the pseudocontractive mapping $T=I-A$. It is well known that if $A$ is accretive, then the solutions of the equation $0 \in A x$ correspond to the equilibrium points of some evolution systems. For this reason, iterative methods for approximating the zeros of accretive operator $A$ have extensively been studies over the last forty years (see, e.g., [1, 2, 3, 4, 5, 6, 13, 14, 15, 20, 22, 23, 24, 31]).

Let $C$ be a closed convex subset of $E$ and $T: C \rightarrow C$ a nonexpansive mapping. In [16], Kirk studied the iterative scheme given by

$$
x_{n+1}=a_{0} x_{n}+a_{1} T x_{n}+a_{2} T^{2} x_{n}+\cdots+a_{k} T^{k} x_{n}, \quad n \geq 0,
$$

where $x_{0} \in C, a_{i} \geq 0, a_{0}>0$ and $\sum_{i=0}^{k} a_{i}=1$ for approximating fixed points of nonexpansive mappings. Liu et al. [18] introduced the following iterative scheme for finite nonexpansive mappings $T_{i}: C \rightarrow C(i=1, \ldots, k)$ :

$$
x_{n+1}=a_{0} x_{n}+a_{1} T_{1} x_{n}+a_{2} T_{2} x_{n}+\cdots+a_{k} T_{k} x_{n}, \quad n \geq 0,
$$

where $x_{0} \in C, a_{i} \geq 0, a_{0}>0$ and $\sum_{i=0}^{k} a_{i}=1$, and showed that $\left\{x_{n}\right\}$ generated by (1.1) converges to a common fixed point of $T_{i}(i=1,2, \ldots, k)$, in a Banach space with a certain property, say, condition A. The result improved the corresponding result of Kirk [16], Maiti and Saha [19] and Senter and Doston [25]. In 2002, Jung [9] established the weak convergence of $\left\{x_{n}\right\}$ generated by (1.1) in a reflexive and strictly convex Banach space having a uniformly Gâteaux differentiable norm.

Recently, Zegeye and Shahzad [32] considered the following iterative scheme for a finite family of $m$-accretive operators $A_{i}: C \rightarrow E(i=1, \ldots, k)$ :

$$
x_{n+1}=\alpha_{n} u+\left(1-\alpha_{n}\right) S_{k} x_{n}, n \geq 0,
$$

where $S_{k}:=a_{0} I+a_{1} J_{A_{1}}+a_{2} J_{A_{2}}+\cdots+a_{k} J_{A_{k}}$ with $J_{A_{i}}:\left(I+A_{i}\right)^{-1}$ for $0<a_{i}<1(i=0,1, \ldots, k), \sum_{i=0}^{k} a_{i}=1$, and under the control conditions:

(i) $\lim _{n \rightarrow \infty} \alpha_{n}=0$, 
(ii) $\sum_{n=0}^{\infty} \alpha_{n}=\infty$, or, equivalently, $\prod_{n=0}^{\infty}\left(1-\alpha_{n}\right)=0$,

(iii) $\sum_{n=0}^{\infty}\left|\alpha_{n+1}-\alpha_{n}\right|<\infty$, or (iii) $\lim _{n \rightarrow \infty} \frac{\left|\alpha_{n+1}-\alpha_{n}\right|}{\alpha_{n+1}}=0$,

showed that the sequence $\left\{x_{n}\right\}$ generated by (1.2) converges strongly to a common solution of the equation $A_{i} x \ni 0$ for $i=1, \ldots, k$ in a reflexive and strictly convex Banach space having a uniformly Gâteaux differentiable norm and satisfying that every weakly compact convex subset of $E$ has the fixed point property for nonexpansive mapping. On the other hand, as the viscosity approximation method, Moudafi [21] and $\mathrm{Xu}$ [30] considered the iterative scheme: for $T$ a nonexpansive mapping, $f \in \Sigma_{C}$ and $\alpha_{n} \in(0,1)$,

$$
x_{n+1}=\alpha_{n} f\left(x_{n}\right)+\left(1-\alpha_{n}\right) T x_{n}, \quad n \geq 0 .
$$

Under the conditions (i), (ii) and (iii) on $\left\{\alpha_{n}\right\}, \mathrm{Xu}[30]$ showed in a uniformly smooth Banach space that the sequence $\left\{x_{n}\right\}$ generated by (1.3) converges strongly to a fixed point of $T$, which solves a certain variational inequality. The results of $\mathrm{Xu}$ [30] extended the results of Moudafi [21] to a Banach space setting. In 2006, Jung [10] considered the iterative scheme: for $N>1, T_{1}, T_{2}, \ldots, T_{k}$ nonexpansive mappings, $f \in \Sigma_{C}$ and $\alpha_{n} \in(0,1)$,

$$
x_{n+1}=\alpha_{n} f\left(x_{n}\right)+\left(1-\alpha_{n}\right) T_{n+1} x_{n}, \quad n \geq 0,
$$

where $T_{n}:=T_{n} \bmod k$, and extended results of Xu [30] (and Moudafi [21]) to the case of a family of finite nonexpansive mappings. In particular, under the conditions (i), (ii) and the perturbed control condition on $\left\{\alpha_{n}\right\}$

(iv) $\left|\alpha_{n+k}-\alpha_{n}\right| \leq o\left(\alpha_{n+k}\right)+\sigma_{n}, \quad \sum_{n=0}^{\infty} \sigma_{n}<\infty$,

he obtained the strong convergence of the sequence $\left\{x_{n}\right\}$ generated by (1.4) to a solution in $\bigcap_{i=1}^{k} \operatorname{Fix}\left(T_{i}\right)$ of a certain variational inequality in a reflexive Banach space having a uniformly Gâteaux differentiable norm with the assumption that every weakly compact convex subset of $E$ has the fixed point property for nonexpansive mapping, and gave an example which satisfies the conditions (i), (ii) and (iv), but fails to satisfy the condition (iii) for $k>1 ; \sum_{n=0}^{\infty}\left|\alpha_{n+k}-\alpha_{n}\right|<$ $\infty$.

In this paper, motivated by above-mentioned results, we introduce the viscosity approximation method for a finite family of accretive operators: for resolvent $J_{r_{i}}$ of accretive operator $A_{i}$ such that $\bigcap_{i=1}^{k} N\left(A_{i}\right) \neq \emptyset$ and $\overline{D(A)} \subset$ $C \subset \bigcap_{r>0} R\left(I+r A_{i}\right)(i=1, \ldots, k), f \in \Sigma_{C}$ and $\left\{\alpha_{n}\right\},\left\{\beta_{n}\right\} \subset(0,1)$,

$$
\left\{\begin{array}{l}
x_{0}=x \in C \\
y_{n}=\beta_{n} x_{n}+\left(1-\beta_{n}\right) S_{k} x_{n}, \\
x_{n+1}=\alpha_{n} f\left(x_{n}\right)+\left(1-\alpha_{n}\right) y_{n}, \quad n \geq 0
\end{array}\right.
$$

where $S_{k}:=a_{0} I+a_{1} J_{r_{1}}^{A_{1}}+a_{2} J_{r_{2}}^{A_{2}}+\cdots+a_{k} J_{r_{k}}^{A_{k}}$ with $J_{r_{i}}^{A_{i}}:=\left(I+r_{i} A_{i}\right)^{-1}$ for $r_{i}>0$, and $0<a_{i}<1(i=0,1, \ldots, k)$ and $\sum_{i=0}^{k} a_{i}=1$, and establish the strong convergence of the sequence $\left\{x_{n}\right\}$ generated by (IS) to a common solution of the equations $A_{i} x \ni 0$ for $i=1, \ldots, k$, in a reflexive and strictly 
convex Banach space having a uniformly Gâteaux differentiable norm under certain different control conditions on sequences $\left\{\alpha_{n}\right\}$ and $\left\{\beta_{n}\right\}$. The main results improve the recent results of Wong et al. [29] and Zegeye and Shahzad [32]. Our results also improve the corresponding results of $[9,16,18,19,25]$ for finite nonexpansive mappings to the case of finite pseudocontractive mappings.

\section{Preliminaries and lemmas}

Let $E$ be a real Banach space with norm $\|\cdot\|$ and let $E^{*}$ be its dual. The value of $f \in E^{*}$ at $x \in E$ will be denoted by $\langle x, f\rangle$. When $\left\{x_{n}\right\}$ is a sequence in $E$, then $x_{n} \rightarrow x$ (resp., $x_{n} \rightarrow x, x_{n} \stackrel{*}{\rightarrow} x$ ) will denote strong (resp., weak, weak $^{*}$ ) convergence of the sequence $\left\{x_{n}\right\}$ to $x$.

The norm of $E$ is said to be Gâteaux differentiable if

$$
\lim _{t \rightarrow 0} \frac{\|x+t y\|-\|x\|}{t}
$$

exists for each $x, y$ in its unit sphere $U=\{x \in E:\|x\|=1\}$. Such an $E$ is called a smooth Banach space. The norm is said to be uniformly Gâteaux differentiable if for $y \in U$, the limit is attained uniformly for $x \in U$. The space $E$ is said to have a uniformly Fréchet differentiable norm (and $E$ is said to be uniformly smooth) if the limit in (2.1) is attained uniformly for $(x, y) \in U \times U$. It is well known that if $E$ has a uniformly Gâteaux differentiable norm, $J$ is uniformly norm to weak* continuous on each bounded subsets of $E([7,28])$.

The (normalized) duality mapping $J$ from $E$ into the family of nonempty (by Hahn-Banach theorem) weak* compact subsets of its dual $E^{*}$ is defined by

$$
J(x)=\left\{f \in E^{*}:\langle x, f\rangle=\|x\|^{2}=\|f\|^{2}\right\}
$$

for each $x \in E$. It is single valued if and only if $E$ is smooth.

A Banach space $E$ is said to be strictly convex if $\left\|a_{1} x_{1}+a_{2} x_{2}+\cdots+a_{k} x_{k}\right\|<1$ for $x_{i} \in E(i=1,2, \ldots, k)$ with $\left\|x_{i}\right\|=1(i=1,2, \ldots, k)$ and $x_{i} \neq x_{j}$ for some $i \neq j$, and for $a_{i} \in(0,1)(i=1,2, \ldots, k)$ such that $\sum_{i=1}^{k} a_{i}=1$.

Let $D$ be a subset of $C$. Then $Q: C \rightarrow D$ is called a retraction from $C$ onto $D$ if $Q x=x$ for all $x \in D$. A retraction $Q: C \rightarrow D$ is said to be sunny if $Q(Q x+t(x-Q x))=Q x$ for all $x \in C$ and $t \geq 0$ whenever $x+t(x-Q x) \in C$. A subset $D$ of $C$ is said to be a sunny nonexpansive retract of $C$ if there exists a sunny nonexpansive retraction of $C$ onto $D$ for more details, see [8]. In a smooth Banach space $E$, it is known [8, p. 48]) that $Q: C \rightarrow D$ is a sunny nonexpansive retraction if and only if the following condition holds:

$$
\langle x-Q x, J(z-Q x)\rangle \leq 0, \quad x \in C, \quad z \in D .
$$

We need the following lemmas for the proof of our main results. Lemma 2.1 was also given in [11]. Lemma 2.2 is Lemma 2 of [27] and Lemma 2.3 is essentially Lemma 2 of [17]. 
Lemma 2.1. Let $X$ be a real Banach space and $J$ be the duality mapping. Then, for any given $x, y \in X$, one has

$$
\|x+y\|^{2} \leq\|x\|^{2}+2\langle y, j(x+y)\rangle
$$

for all $j(x+y) \in J(x+y)$.

Lemma 2.2. Let $\left\{x_{n}\right\}$ and $\left\{w_{n}\right\}$ be bounded sequences in a Banach space $E$ and let $\left\{\gamma_{n}\right\}$ be a sequence in $[0,1]$ which satisfies the following condition:

$$
0<\liminf _{n \rightarrow \infty} \gamma_{n} \leq \limsup _{n \rightarrow \infty} \gamma_{n}<1 .
$$

Suppose that $x_{n+1}=\gamma_{n} x_{n}+\left(1-\gamma_{n}\right) w_{n}, n \geq 0$, and

$$
\limsup _{n \rightarrow \infty}\left(\left\|w_{n+1}-w_{n}\right\|-\left\|x_{n+1}-x_{n}\right\|\right) \leq 0 .
$$

Then $\lim _{n \rightarrow \infty}\left\|w_{n}-x_{n}\right\|=0$.

Lemma 2.3. Let $\left\{s_{n}\right\}$ be a sequence of non-negative real numbers satisfying

$$
s_{n+1} \leq\left(1-\lambda_{n}\right) s_{n}+\lambda_{n} \delta_{n}+\gamma_{n}, \quad n \geq 0,
$$

where $\left\{\lambda_{n}\right\},\left\{\delta_{n}\right\}$ and $\left\{\gamma_{n}\right\}$ satisfy the following conditions:

(i) $\left\{\lambda_{n}\right\} \subset[0,1]$ and $\sum_{n=0}^{\infty} \lambda_{n}=\infty$;

(ii) $\limsup _{n \rightarrow \infty} \delta_{n} \leq 0$ or $\sum_{n=1}^{\infty} \lambda_{n} \delta_{n}<\infty$;

(iii) $\gamma_{n} \geq 0(n \geq 0), \sum_{n=0}^{\infty} \gamma_{n}<\infty$.

Then $\lim _{n \rightarrow \infty} s_{n}=0$.

By using the same method as Lemma 3.1 in [32], we can prove the following lemma. So we omit its proof.

Lemma 2.4. Let $E$ be a strictly convex Banach space. Let $C$ be a nonempty closed convex subset of $E$ and $A_{i} \subset E \times E(i=1, \ldots, k)$ accretive operators in $E$ such that $\bigcap_{i=1}^{k} N\left(A_{i}\right) \neq \emptyset$ and $\overline{D\left(A_{i}\right)} \subset C \subset \bigcap_{r>0} R\left(I+r A_{i}\right)$. Let $S_{k}:=$ $a_{0} I+a_{1} J_{r_{1}}^{A_{1}}+\cdots+a_{k} J_{r_{k}}^{A_{k}}$ with $J_{r_{i}}^{A_{i}}:=\left(I+r_{i} A_{i}\right)^{-1}$ for $r_{i}>0(i=1, \ldots, k)$, $0<a_{i}<1(i=0,1, \ldots, k)$ and $\sum_{i=0}^{k} a_{i}=1$. Then $S_{k}$ is nonexpansive and $F\left(S_{k}\right)=\bigcap_{i=1}^{k} N\left(A_{i}\right)$.

\section{Main results}

Now, we study the strong convergence results for the iterative scheme (IS) in Banach spaces.

We need the following result for the existence of a solution of the variational inequality

$$
\langle(I-f)(q), J(q-p)\rangle \leq 0, \quad f \in \Sigma_{C}, \quad p \in F(T),
$$

which Jung and Sahu [12] established recently.

Theorem JS. ([12, Theorem 2]) Let $E$ be a reflexive and strictly convex Banach space having a uniformly Gâteaux differentiable norm, $C$ a nonempty closed convex subset of $E, A: C \rightarrow C$ a continuous strongly pseudocontractive 
mapping with constant $k \in[0,1)$ and $T: C \rightarrow E$ a continuous pseudocontractive mapping satisfying the weakly inward condition. If $T$ has a fixed point in $C$, then the path $\left\{x_{t}\right\}$ defined by

$$
x_{t}=t A x_{t}+(1-t) T x_{t}, \quad t \in(0,1)
$$

converges strongly to a fixed point $q$ of $T$, which is the unique solution of a variational inequality:

$$
\langle(I-A) q, J(q-p)\rangle \leq 0 \text { for all } p \in F(T) .
$$

Remark 3.1. (1) Theorem JS generalizes Theorem 3.1 of Song and Chen [26] to a more general class of mappings. In fact, in Theorem 3.1 of [26], $T: C \rightarrow C$ is a nonexpansive self-mapping and $A=f$ is a contraction.

(2) In Theorem JS, if $A(x)=u, x \in C$, is a constant and $Q u=q=$ $\lim _{t \rightarrow 0} x_{t}$, then it follows from $(2.2)$ that $Q$ is reduced to the sunny nonexpansive retraction from $C$ onto $F(T)$,

$$
\langle Q u-u, J(Q u-p)\rangle \leq 0, \quad u \in C, \quad p \in F(T) .
$$

Using Theorem JS, we establish the following main result.

Theorem 3.1. Let $E$ be a reflexive and strictly convex Banach space having a uniformly Gâteaux differentiable norm. Let $C$ be a nonempty closed convex subset of $E$ and $A_{i} \subset E \times E(i=1, \ldots, k)$ accretive operators in $E$ such that $\bigcap_{i=1}^{k} N\left(A_{i}\right) \neq \emptyset$ and $\overline{D\left(A_{i}\right)} \subset C \subset \bigcap_{r>0} R\left(I+r A_{i}\right)$. Let $\left\{\alpha_{n}\right\}$ and $\left\{\beta_{n}\right\}$ be sequences in $(0,1)$ which satisfy the conditions:

(C1) $\lim _{n \rightarrow \infty} \alpha_{n}=0$;

(C2) $\sum_{n=0}^{\infty} \alpha_{n}=\infty$;

(C3) $0<\liminf _{n \rightarrow \infty} \beta_{n} \leq \limsup _{n \rightarrow \infty} \beta_{n}<1$.

Let $f \in \Sigma_{C}$ and $x_{0} \in C$ be chosen arbitrarily. Let $\left\{x_{n}\right\}$ be a sequence generated by

$$
\left\{\begin{array}{l}
x_{0}=x \in C \\
y_{n}=\beta_{n} x_{n}+\left(1-\beta_{n}\right) S_{k} x_{n}, \\
x_{n+1}=\alpha_{n} f\left(x_{n}\right)+\left(1-\alpha_{n}\right) y_{n}
\end{array}\right.
$$

where $S_{k}:=a_{0} I+a_{1} J_{r_{1}}^{A_{1}}+\cdots+a_{k} J_{r_{k}}^{A_{k}}$ with $J_{r_{i}}^{A_{i}}:=\left(I+r_{i} A_{i}\right)^{-1}$ for $r_{i}>0$ $(i=1, \ldots, k), 0<a_{i}<1(i=0,1, \ldots, k)$ and $\sum_{i=0}^{k} a_{i}=1$. Then $\left\{x_{n}\right\}$ converges strongly to $q \in F\left(S_{k}\right)=\bigcap_{i=1}^{k} N\left(A_{i}\right)$, where $q$ is the unique solution of the variational inequality

$$
\langle(I-f)(q), J(q-p)\rangle \leq 0, \quad f \in \Sigma_{C}, \quad p \in F\left(S_{k}\right) .
$$


Proof. First, we note that by Theorem JS and Lemma 2.4, there exists the unique solution $q \in F\left(S_{k}\right)=\bigcap_{i=1}^{k} N\left(A_{i}\right)$ of the variational inequality

$$
\langle(I-f)(q), J(q-p)\rangle \leq 0, \quad f \in \Sigma_{C}, \quad p \in F\left(S_{k}\right),
$$

where $q=\lim _{t \rightarrow 0} z_{t}$ and $z_{t}$ is defined by $z_{t}=t f\left(z_{t}\right)+(1-t) S_{k} z_{t}$ for $0<t<1$.

We proceed with the following steps:

Step 1. We show that $\left\|x_{n}-p\right\| \leq \max \left\{\left\|x_{0}-p\right\|, \frac{1}{1-k}\|f(p)-p\|\right\}$ for all $n \geq 0$ and all $p \in F$ and so $\left\{x_{n}\right\}$ is bounded. Indeed, let $p \in F\left(S_{k}\right)$ and $d=\max \left\{\left\|x_{0}-p\right\|, \frac{1}{1-k}\|f(p)-p\|\right\}$. Noting that

$$
\left\|y_{n}-p\right\| \leq \beta_{n}\left\|x_{n}-p\right\|+\left(1-\beta_{n}\right)\left\|S_{k} x_{n}-p\right\| \leq\left\|x_{n}-p\right\|,
$$

we have

$$
\begin{aligned}
\left\|x_{1}-p\right\| & \leq\left(1-\alpha_{0}\right)\left\|y_{0}-p\right\|+\alpha_{0}\left\|f\left(x_{0}\right)-p\right\| \\
& \leq\left(1-\alpha_{0}\right)\left\|x_{0}-p\right\|+\alpha_{0}\left(\left\|f\left(x_{0}\right)-f(p)\right\|+\|f(p)-p\|\right) \\
& \leq\left(1-(1-k) \alpha_{0}\right)\left\|x_{0}-p\right\|+\alpha_{0}\|f(p)-p\| \\
& \leq\left(1-(1-k) \alpha_{0}\right) d+\alpha_{0}(1-k) d=d .
\end{aligned}
$$

Using an induction, we obtain $\left\|x_{n+1}-p\right\| \leq d$. Hence $\left\{x_{n}\right\}$ is bounded, and so are $\left\{y_{n}\right\},\left\{S_{k} x_{n}\right\}$ and $\left\{f\left(x_{n}\right)\right\}$.

Step 2. We show that $\lim _{n \rightarrow \infty}\left\|x_{n+1}-x_{n}\right\|$. To this end, set $\gamma_{n}=(1-$ $\left.\alpha_{n}\right) \beta_{n}, \quad n \geq 0$. Then it follow from (C1)and (C3) that

$$
0<\liminf _{n \rightarrow \infty} \gamma_{n} \leq \limsup _{n \rightarrow \infty} \gamma_{n}<1 \text {. }
$$

Define

$$
x_{n+1}=\gamma_{n} x_{n}+\left(1-\gamma_{n}\right) w_{n} \text {. }
$$

Observe that

$$
\begin{aligned}
w_{n+1}-w_{n}= & \frac{x_{n+2}-\gamma_{n+1} x_{n+1}}{1-\gamma_{n+1}}-\frac{x_{n+1}-\gamma_{n} x_{n}}{1-\gamma_{n}} \\
= & \frac{\alpha_{n+1} f\left(x_{n+1}\right)+\left(1-\alpha_{n+1}\right) y_{n+1}-\gamma_{n+1} x_{n+1}}{1-\gamma_{n+1}} \\
& -\frac{\alpha_{n} f\left(x_{n}\right)+\left(1-\alpha_{n}\right) y_{n}-\gamma_{n} x_{n}}{1-\gamma_{n}} \\
= & \left(\frac{\alpha_{n+1} f\left(x_{n+1}\right)}{1-\gamma_{n+1}}-\frac{\alpha_{n} f\left(x_{n}\right)}{1-\gamma_{n}}\right) \\
& -\frac{\left(1-\alpha_{n}\right)\left[\beta_{n} x_{n}+\left(1-\beta_{n}\right) S_{k} x_{n}\right]-\gamma_{n} x_{n}}{1-\gamma_{n}} \\
& +\frac{\left(1-\alpha_{n+1}\right)\left[\beta_{n+1} x_{n+1}+\left(1-\beta_{n+1}\right) S_{k} x_{n+1}\right]-\gamma_{n+1} x_{n+1}}{1-\gamma_{n+1}} \\
= & \left(\frac{\alpha_{n+1} f\left(x_{n+1}\right)}{1-\gamma_{n+1}}-\frac{\alpha_{n} f\left(x_{n}\right)}{1-\gamma_{n}}\right)+\frac{\left(1-\alpha_{n+1}\right)\left(1-\beta_{n+1}\right) S_{k} x_{n+1}}{1-\gamma_{n+1}}
\end{aligned}
$$




$$
\begin{aligned}
& -\frac{\left(1-\alpha_{n}\right)\left(1-\beta_{n}\right) S_{k} x_{n}}{1-\gamma_{n}} \\
= & \left(\frac{\alpha_{n+1} f\left(x_{n+1}\right)}{1-\gamma_{n+1}}-\frac{\alpha_{n} f\left(x_{n}\right)}{1-\gamma_{n}}\right)+\left(S_{k} x_{n+1}-S_{k} x_{n}\right) \\
& -\frac{\alpha_{n+1}}{1-\gamma_{n+1}} S_{k} x_{n+1}+\frac{\alpha_{n}}{1-\gamma_{n}} S_{k} x_{n} .
\end{aligned}
$$

It follows from (3.3) that

$$
\begin{aligned}
& \left\|w_{n+1}-w_{n}\right\|-\left\|x_{n+1}-x_{n}\right\| \\
\leq & \frac{\alpha_{n+1}}{1-\gamma_{n+1}}\left(\left\|f\left(x_{n+1}\right)\right\|+\left\|S_{k} x_{n+1}\right\|\right)+\frac{\alpha_{n}}{1-\gamma_{n}}\left(\left\|f\left(x_{n}\right)\right\|+\left\|S_{k} x_{n}\right\|\right) .
\end{aligned}
$$

Since $\left\{f\left(x_{n}\right)\right\}$ and $\left\{S_{k} x_{n}\right\}$ are bounded, by (C1), (3.1) and (3.4) we obtain that

$$
\limsup _{n \rightarrow \infty}\left(\left\|w_{n+1}-w_{n}\right\|-\left\|x_{n+1}-x_{n}\right\|\right) \leq 0 .
$$

Hence by Lemma 2.2, we have

$$
\lim _{n \rightarrow \infty}\left\|w_{n}-x_{n}\right\|=0
$$

It then follows from (3.1) and (3.2) that

$$
\lim _{n \rightarrow \infty}\left\|x_{n+1}-x_{n}\right\|=0 \text {. }
$$

Step 3. We show that $\lim _{n \rightarrow \infty}\left\|x_{n}-S_{k} x_{n}\right\|=0$. Indeed, as a consequence with the control condition (C1), by Step 1, we get

$$
\left\|x_{n+1}-y_{n}\right\| \leq \alpha_{n}\left(\left\|f\left(x_{n}\right)\right\|+\left\|y_{n}\right\|\right) \rightarrow 0 \quad(n \rightarrow \infty) .
$$

Combining Step 2 and (3.6), we get

$$
\lim _{n \rightarrow \infty}\left\|x_{n}-y_{n}\right\|=0
$$

Observe that

$$
y_{n}-x_{n}=\left(1-\beta_{n}\right)\left(S_{k} x_{n}-x_{n}\right) .
$$

It follows from (C3), (3.7) and (3.8)

$$
\lim _{n \rightarrow \infty}\left\|x_{n}-S_{k} x_{n}\right\|=0 .
$$

Step 4. We show that $\lim \sup _{n \rightarrow \infty}\left\langle(I-f)(q), J\left(q-x_{n}\right)\right\rangle \leq 0$. To prove this, let a subsequence $\left\{x_{n_{j}}\right\}$ of $\left\{x_{n}\right\}$ be such that

$$
\limsup _{n \rightarrow \infty}\left\langle(I-f)(q), J\left(q-x_{n}\right)\right\rangle=\lim _{j \rightarrow \infty}\left\langle(I-f)(q), J\left(q-x_{n_{j}}\right)\right\rangle
$$

and

$$
x_{n_{j}} \rightarrow p \quad \text { for some } p \in E \text {. }
$$

Now let $z_{t}$ be defined by $z_{t}=t f\left(z_{t}\right)+(1-t) S_{k} z_{t}$ for $0<t<1$. Then

$$
z_{t}-x_{n}=(1-t)\left(S_{k} z_{t}-x_{n}\right)+t\left(f\left(z_{t}\right)-x_{n}\right) .
$$


Applying Lemma 2.1, we have

$$
\left\|z_{t}-x_{n}\right\|^{2} \leq(1-t)^{2}\left\|S_{k} z_{t}-x_{n}\right\|^{2}+2 t\left\langle f\left(z_{t}\right)-x_{n}, J\left(z_{t}-x_{n}\right)\right\rangle .
$$

Putting

$$
a_{j}(t)=(1-t)^{2}\left\|S_{k} x_{n_{j}}-x_{n_{j}}\right\|\left(2\left\|z_{t}-x_{n_{j}}\right\|+\left\|S_{k} x_{n_{j}}-x_{n_{j}}\right\|\right) \rightarrow 0(j \rightarrow \infty)
$$

by Step 3 and using Lemma 2.1, we obtain

$$
\begin{aligned}
\left\|z_{t}-x_{n_{j}}\right\|^{2} \leq & (1-t)^{2}\left\|S_{k} z_{t}-x_{n_{j}}\right\|^{2}+2 t\left\langle f\left(z_{t}\right)-x_{n_{j}}, J\left(z_{t}-x_{n_{j}}\right)\right\rangle \\
\leq & (1-t)^{2}\left(\left\|S_{k} z_{t}-S_{k} x_{n_{j}}\right\|+\left\|S_{k} x_{n_{j}}-x_{n_{j}}\right\|\right)^{2} \\
& \quad+2 t\left\langle f\left(z_{t}\right)-z_{t}, J\left(z_{t}-x_{n_{j}}\right)\right\rangle+2 t\left\|z_{t}-x_{n_{j}}\right\|^{2} \\
\leq & (1-t)^{2}\left\|z_{t}-x_{n_{j}}\right\|^{2}+a_{j}(t) \\
& \quad+2 t\left\langle f\left(z_{t}\right)-z_{t}, J\left(z_{t}-x_{n_{j}}\right)\right\rangle+2 t\left\|z_{t}-x_{n_{j}}\right\|^{2} .
\end{aligned}
$$

The last inequality implies

$$
\left\langle z_{t}-f\left(z_{t}\right), J\left(z_{t}-x_{n_{j}}\right)\right\rangle \leq \frac{t}{2}\left\|z_{t}-x_{n_{j}}\right\|^{2}+\frac{1}{2 t} a_{j}(t)
$$

It follows that

$$
\lim _{j \rightarrow \infty}\left\langle z_{t}-f\left(z_{t}\right), J\left(z_{t}-x_{n_{j}}\right)\right\rangle \leq \frac{t}{2} M
$$

where $M>0$ is a constant such that $M \geq\left\|z_{t}-x_{n}\right\|^{2}$ for all $n \geq 0$ and $t \in(0,1)$. Taking the limsup as $t \rightarrow 0$ in (3.9) and noticing the fact that the two limits are interchangeable due to the fact that the duality mapping $J$ is norm to weak* uniformly continuous on bounded subset of $E$, we have

$$
\limsup _{j \rightarrow \infty}\left\langle(I-f)(q), J\left(q-x_{n_{j}}\right)\right\rangle \leq 0 .
$$

Indeed, letting $t \rightarrow 0$, from (3.9) we have

$$
\limsup _{t \rightarrow 0} \limsup _{j \rightarrow \infty}\left\langle z_{t}-f\left(z_{t}\right), J\left(z_{t}-x_{n_{j}}\right)\right\rangle \leq 0 .
$$

So, for any $\varepsilon>0$, there exists a positive number $\delta_{1}$ such that for any $t \in\left(0, \delta_{1}\right)$,

$$
\limsup _{j \rightarrow \infty}\left\langle z_{t}-f\left(z_{t}\right), J\left(z_{t}-x_{n_{j}}\right)\right\rangle \leq \frac{\varepsilon}{2} .
$$

Moreover, since $z_{t} \rightarrow q$ as $t \rightarrow 0$, the set $\left\{z_{t}-x_{n_{j}}\right\}$ is bounded and the duality mapping $J$ is norm to weak* uniformly continuous on bounded subset of $E$, there exists $\delta_{2}>0$ such that, for any $t \in\left(0, \delta_{2}\right)$,

$$
\begin{aligned}
& \left|\left\langle q-f(q), J\left(q-x_{n_{j}}\right)\right\rangle-\left\langle z_{t}-f\left(z_{t}\right), J\left(z_{t}-x_{n_{j}}\right)\right\rangle\right| \\
= & \left|\left\langle q-f(q), J\left(q-x_{n_{j}}\right)-J\left(z_{t}-x_{n_{j}}\right)\right\rangle+\left\langle q-f(q)-\left(z_{t}-f\left(z_{t}\right)\right), J\left(z_{t}-x_{n_{j}}\right)\right\rangle\right| \\
\leq & \left|\left\langle q-f(q), J\left(z_{t}-x_{n_{j}}\right)-J\left(q-x_{n_{j}}\right)\right\rangle\right|+\left\|q-f(q)-\left(z_{t}-f\left(z_{t}\right)\right)\right\|\left\|z_{t}-x_{n_{j}}\right\| \\
< & \frac{\varepsilon}{2} .
\end{aligned}
$$


Choose $\delta=\min \left\{\delta_{1}, \delta_{2}\right\}$, we have for all $t \in(0, \delta)$ and $j \in \mathbb{N}$,

$$
\left\langle q-f(q), J\left(q-x_{n_{j}}\right)\right\rangle\left\langle\left\langle z_{t}-f\left(z_{t}\right), J\left(z_{t}-x_{n_{j}}\right)\right\rangle+\frac{\varepsilon}{2},\right.
$$

which implies that

$$
\limsup _{j \rightarrow \infty}\left\langle q-f(q), J\left(q-x_{n_{j}}\right)\right\rangle \leq \limsup _{j \rightarrow \infty}\left\langle z_{t}-f\left(z_{t}\right), J\left(z_{t}-x_{n_{j}}\right)\right\rangle+\frac{\varepsilon}{2} .
$$

Since $\lim \sup _{j \rightarrow \infty}\left\langle z_{t}-f\left(z_{t}\right), J\left(z_{t}-x_{n_{j}}\right)\right\rangle \leq \frac{\varepsilon}{2}$, we have

$$
\limsup _{j \rightarrow \infty}\left\langle q-f(q), J\left(q-x_{n_{j}}\right)\right\rangle \leq \varepsilon .
$$

Since $\varepsilon$ is arbitrary, we obtain that

$$
\limsup _{j \rightarrow \infty}\left\langle(I-f)(q), J\left(q-x_{n_{j}}\right)\right\rangle \leq 0 .
$$

Step 5. We show that $\lim _{n \rightarrow \infty}\left\|x_{n}-q\right\|=0$. By using (IS), we have

$$
x_{n+1}-q=\alpha_{n}\left(f\left(x_{n}\right)-q\right)+\left(1-\alpha_{n}\right)\left(y_{n}-q\right) .
$$

Applying Lemma 2.1, we obtain

$$
\begin{aligned}
\left\|x_{n+1}-q\right\|^{2} \leq & \left(1-\alpha_{n}\right)^{2}\left\|y_{n}-q\right\|^{2}+2 \alpha_{n}\left\langle f\left(x_{n}\right)-q, J\left(x_{n+1}-q\right)\right\rangle \\
\leq & \left(1-\alpha_{n}\right)^{2}\left\|x_{n}-q\right\|^{2}+2 \alpha_{n}\left\langle f\left(x_{n}\right)-f(q), J\left(x_{n+1}-q\right)\right\rangle \\
& +2 \alpha_{n}\left\langle f(q)-q, J\left(x_{n+1}-q\right)\right\rangle \\
\leq & \left(1-\alpha_{n}\right)^{2}\left\|x_{n}-q\right\|^{2}+2 k \alpha_{n}\left\|x_{n}-q\right\|\left\|x_{n+1}-q\right\| \\
& +2 \alpha_{n}\left\langle f(q)-q, J\left(x_{n+1}-q\right)\right\rangle \\
\leq & \left(1-\alpha_{n}\right)^{2}\left\|x_{n}-q\right\|^{2}+k \alpha_{n}\left(\left\|x_{n}-q\right\|^{2}+\left\|x_{n+1}-q\right\|^{2}\right) \\
& +2 \alpha_{n}\left\langle f(q)-q, J\left(x_{n+1}-q\right)\right\rangle .
\end{aligned}
$$

It then follows that

$$
\begin{aligned}
\left\|x_{n+1}-q\right\|^{2} \leq & \frac{1-(2-k) \alpha_{n}+\alpha_{n}^{2}}{1-k \alpha_{n}}\left\|x_{n}-q\right\|^{2} \\
& +\frac{2 \alpha_{n}}{1-k \alpha_{n}}\left\langle f(q)-q, J\left(x_{n+1}-q\right)\right\rangle \\
\leq & \frac{1-(2-k) \alpha_{n}}{1-k \alpha_{n}}\left\|x_{n}-q\right\|^{2}+\frac{\alpha_{n}^{2}}{1-k \alpha_{n}} M \\
& +\frac{2 \alpha_{n}}{1-k \alpha_{n}}\left\langle(I-f)(q), J\left(q-x_{n+1}\right)\right\rangle
\end{aligned}
$$

where $M=\sup _{n \geq 0}\left\|x_{n}-q\right\|^{2}$. Put

$$
\begin{aligned}
\lambda_{n} & =\frac{2(1-k) \alpha_{n}}{1-k \alpha_{n}} \text { and } \\
\delta_{n} & =\frac{M \alpha_{n}}{2(1-k)}+\frac{1}{1-k}\left\langle(I-f)(q), J\left(q-x_{n+1}\right)\right\rangle .
\end{aligned}
$$


From (C1), (C2) and Step 4, it follows that $\lambda_{n} \rightarrow 0, \sum_{n=0}^{\infty} \lambda_{n}=\infty$ and $\lim \sup _{n \rightarrow \infty} \delta_{n} \leq 0$. Since (3.10) reduces to

$$
\left\|x_{n+1}-q\right\|^{2} \leq\left(1-\lambda_{n}\right)\left\|x_{n}-q\right\|^{2}+\lambda_{n} \delta_{n},
$$

from Lemma 2.3, we conclude that $\lim _{n \rightarrow \infty}\left\|x_{n}-q\right\|=0$.

Corollary 3.1. Let $E$ be a uniformly convex and uniformly smooth Banach space. Let $A_{i}(i=1, \ldots, k)$ be $m$-accretive operators in $E$ such that $C=\overline{D\left(A_{i}\right)}$ is convex and $\bigcap_{i=1}^{k} N\left(A_{i}\right) \neq \emptyset$. Let $J_{r_{i}}^{A_{i}}(i=1, \ldots, k), S_{k},\left\{\alpha_{n}\right\},\left\{\beta_{n}\right\}, f, x_{0}$ and $\left\{x_{n}\right\}$ be as in Theorem 3.1. Then the conclusion of Theorem 3.1 still holds.

Remark 3.2. (1) Theorem 3.1 supplements Theorem 3.3 of Zegeye and Shahzad [32] in several aspects. In particular, Theorem 3.1 develops Theorem 3.3 of Zegeye and Shahzad [32] to the viscosity method and removes the assumption imposed in Theorem 3.3 of Zegeye and Shahzad [32] that every nonempty closed bounded convex subset of $E$ has the fixed point property for nonexpansive mappings. Moreover, by using the iterative scheme (IS), Theorem 3.1 removes the condition $\sum_{n=0}^{\infty}\left|\alpha_{n+1}-\alpha_{n}\right|<\infty$ or $\lim _{n \rightarrow \infty} \frac{\left|\alpha_{n+1}-\alpha_{n}\right|}{\alpha_{n+1}}$ imposed on sequence $\left\{\alpha_{n}\right\}$ in Theorem 3.3 of Zegeye and Shahzad [32].

(2) Using the iterative scheme (IS), Theorem 3.1 also develops Theorem 6.3 of Wong et al. [29] without the condition $\lim _{n \rightarrow \infty} \frac{\left|\alpha_{n+1}-\alpha_{n}\right|}{\alpha_{n+1}}$.

(3) In general, the conditions (C3) in Theorem 3.1 and the condition

$$
\sum_{n=0}^{\infty}\left|\beta_{n+1}-\beta_{n}\right|<\infty
$$

are not comparable; neither of them implies other.

As a direct consequence of Theorem 3.1, we obtain strong convergence to a common fixed point of a family of pseudocontractive mappings.

Theorem 3.2. Let $E$ be a reflexive and strictly convex Banach space having a uniformly Gâteaux differentiable norm. Let $C$ be a nonempty closed convex subset of $E$ and $T_{i}: C \rightarrow E(i=1, \ldots, k)$ pseudocontractive mappings such that $\left(I-T_{i}\right)$ is $m$-accretive on $C$ with $\bigcap_{i=1}^{k} F\left(T_{i}\right) \neq \emptyset$. Let $J_{T_{i}}:=\left(I+\left(I-T_{i}\right)\right)^{-1}$ $=\left(2 I-T_{i}\right)^{-1}$ for $i=1, \ldots, k$. Let $\left\{\alpha_{n}\right\}$ and $\left\{\beta_{n}\right\}$ be sequences in $(0,1)$ which satisfy the conditions:

(C1) $\lim _{n \rightarrow \infty} \alpha_{n}=0$;

(C2) $\sum_{n=0}^{\infty} \alpha_{n}=\infty$;

(C3) $0<\liminf _{n \rightarrow \infty} \beta_{n} \leq \limsup _{n \rightarrow \infty} \beta_{n}<1$.

Let $f \in \Sigma_{C}$ and $x_{0} \in C$ be chosen arbitrarily. Let $\left\{x_{n}\right\}$ be a sequence generated by

$$
\left\{\begin{array}{l}
x_{0}=x \in C \\
y_{n}=\beta_{n} x_{n}+\left(1-\beta_{n}\right) S_{k} x_{n} \\
x_{n+1}=\alpha_{n} f\left(x_{n}\right)+\left(1-\alpha_{n}\right) y_{n}, \quad n \geq 0
\end{array}\right.
$$


where $S_{k}:=a_{0} I+a_{1} J_{T_{1}}+\cdots+a_{k} J_{T_{k}}$ for $0<a_{i}<1(i=0,1, \ldots, k)$ and $\sum_{i=0}^{k} a_{i}=1$. Then $\left\{x_{n}\right\}$ converges strongly to $q \in F\left(S_{k}\right)=\bigcap_{i=1}^{k} F\left(T_{i}\right)$, where $q$ is the unique solution of the variational inequality

$$
\langle(I-f)(q), J(q-p)\rangle \leq 0, \quad f \in \Sigma_{C}, \quad p \in F\left(S_{k}\right) .
$$

Proof. Let $A_{i}:=\left(I-T_{i}\right)$ for each $i=1, \ldots, k$. Then clearly, $F\left(T_{i}\right)=N\left(A_{i}\right)$ and hence $\bigcap_{i=1}^{k} N\left(A_{i}\right)=\bigcap_{i=1}^{k} F\left(T_{i}\right) \neq \emptyset$. Moreover, each $A_{i}$ for $i=1, \ldots, k$ is $m$-accretive. Thus the results follows from Theorem 3.2.

Remark 3.3. (1) Theorem 3.2 complements Theorem 3.9 of Zegeye and Shahzad [32] to the viscosity method together with certain different control conditions in more general Banach space.

(2) Theorem 3.2 also develops the corresponding results of $[9,16,18,19$, 25] for finite nonexpansive mappings to the case of finite pseudocontractive mappings.

(3) We point out that our results are applicable to, in particular, in all $L^{p}$ spaces, $1<p<\infty$.

Acknowledgement. The author would like to thank the anonymous referee for his valuable comments and careful reading of the paper.

\section{References}

[1] K. Aoyama, Y. Kimura, W. Takahashi, and M. Toyoda, Approximation of common fixed points of a countable family of nonexpansive mappings in Banach spaces, Nonlinear Anal. 67 (2007), 2350-2360.

[2] T. D. Benavides, G. L. Acedo, and H. K. Xu, Iterative solutions for zeros of accretive operators, Math. Nach. 248-249 (2003), 62-71.

[3] H. Bréziz and P. L. Lions, Products infinis de resolvents, Israel J. Math. 29 (1978), $329-345$.

[4] R. E. Bruck and G. B. Passty, Almost convergence of the infinite product of resolvents in Banach spaces, Nonlinear Anal. 3 (1979), 279-282.

[5] R. E. Bruck and S. Reich, Nonexpansive projections and resolvents in Banach spaces, Houston J. Math. 3 (1977), 459-470.

[6] R. Chen and Z. Zhu, Viscosity approximation fixed points for nonexpansive and $\mathrm{m}$ acctrive operators, Fixed Point Theory Appl. 2006 (2006), 1-10.

[7] J. Diestel, Geometry of Banach Spaces, Lectures Notes in Math. 485, Springer-Verlag, Berlin, Heidelberg, 1975.

[8] K. Goebel and S. Reich, Uniform Convexity, Hyperbolic Geometry and Nonexpansive Mappings, Marcel Dekker, New York and Basel, 1984.

[9] J. S. Jung, Convergence of nonexpansive iteration process in Banach spaces, J. Math. Anal. Appl. 273 (2002), 153-159.

[10] _ Viscosity approximation methods for a family of finite nonexpansive mappings in Banach spaces, Nonlinear Anal. 64 (2006), 2536-2552.

[11] J. S. Jung and C. Morales, The Mann process for perturbed $m$-accretive operators in Banach spaces, Nonlinear Anal. 46 (2001), 231-243.

[12] J. S. Jung and D. R. Sahu, Convergence of approximating paths to solutions of variational inequalities involving non-Lipschitzian mappings, J. Korean Math. Soc. 45 (2008), no. 2, 377-392. 
[13] J. S. Jung and W. Takahashi, Dual convergence theorems for the nfinite products of resolvents in Banach spaces, Kodai Math. J. 14 (1991), 358-365.

[14] On the asymptotic behavior of infinite products of resolvents in Banach spaces, Nonlinear Anal. 20 (1993), 469-479.

[15] T. H. Kim and H. K. Xu, Strong convergence of modified Mann iterations, Nonlinear Anal. 61 (2005), 51-60.

[16] W. A. Kirk, On successive approximations for nonexpansive mappings in Banach spaces, Glasgow Math. J. 12 (1971), 6-9.

[17] L. S. Liu, Iterative processes with errors for nonlinear strongly accretive mappings in Banach spaces, J. Math. Anal. Appl. 194 (1995), 114-125.

[18] G. Liu, D. Lei, and S. Li, Approximating fixed points of nonexpansive mappings, Internat. J. Math. Math. Sci. 24 (2000), 173-177.

[19] M. Maiti and B. Saha, Approximating fixed points of nonexpansive and generalized nonexpansive mappings, Internat. J. Math. Math. Sci. 16 (1993), 81-86.

[20] H. Miyake and W. Takahashi, Approximating zero points of accretive operators with compact domains in general Banach spaces, Fixed Point Theory Appl. 2005 (2005), no. $1,93-102$.

[21] A. Moudafi, Viscosity approximation methods for fixed-points problems, J. Math. Anal. Appl. 241 (2000), 46-55.

[22] X. Qin and Y. Su, Approximation of a zero point of accretive operator in Banach spaces, J. Math. Anal. Appl. 329 (2007), 415-424.

[23] S. Reich, On infinite products of resolvents, Atti. Accad. Naz. Lincei 63 (1977), 338-340.

[24] _ Weak convergence theorems for nonexpansive mappings in Banach spaces, J. Math. Anal. Appl. 67 (1979), 274-276.

[25] H. F. Senter and W. G. Dotson Jr, Approximating fixed points of nonexpansive mappings, Proc. Amer. Math. Soc. 44 (1974), 375-380.

[26] Y. Song and R. Chen, Strong convergence theorems on an iterative method for a family of finite nonexpansive mappings, Applied. Math. Comput. 180 (2006), 275-287.

[27] T. Suzuki, Strong convergence of Krasnoselskii and Mann's type sequences for one parameter nonexpansive semigroups without Bochner integral, J. Math. Anal. Appl. 305 (2005), 227-239.

[28] W. Takahashi, Nonlinear Functional Analysis, Yokohama Publishers, Yokohama, 2000.

[29] N. C. Wong, D. R. Sahu, and J. C. Yao, Solving variational inequalities involving nonexpansive type mappings, Nonlinear Anal. 69 (2008), 4732-4753.

[30] H. K. Xu, Viscosity approximation methods for nonexpansive mappings, J. Math. Anal. Appl. 298 (2004), 279-291.

[31] _ Strong convergence of an iterative method for nonexpansive and accretive operators, J. Math. Anal. Appl. 314 (2006), 631-643.

[32] H. Zegeye and N. Shahzad, Strong convergence theorems for a common zero of a finite family of accretive operators, Nonlinear Anal. 66 (2007), 1161-1169.

Department of Mathematics

DONG-A UNIVERSITY

Busan 604-714, Korea

E-mail address: jungjs@mail.donga.ac.kr 